\title{
Rudolf, Steffen: Digitale Medien, Partizipation, Ungleichheit. Eine Studie zum sozialen Gebrauch des Internets
}

\author{
Wiesbaden: Springer VS 2019. 394 Seiten. Preis: $€ 39,99$ (e-book)
}

\section{Pablo Porten-Cheé}

Online publiziert: 16. Juli 2020

(C) Der/die Autor(en) 2020

Ungleichheit ist dafür bekannt, sowohl Frust bei den betroffenen Einzelnen als auch öffentlichen Protest auszulösen und gilt als typische Ursache für gesellschaftliche Brüche. Insofern ist Ungleichheit als Kernthema der Sozialwissenschaften auch eines unseres Fachs. Unsere Perspektive auf Ungleichheit ist, und das mag nicht verwundern, wie sozio-strukturelle und individuelle Voraussetzungen den Zugang zu Medien und Informationen prägen und insbesondere hemmen. Im Zuge des Medienwandels werden diese Fragen seit mehreren Jahrzehnten aufgeworfen, wobei die Forschung zum „Digital Divide“ ein wichtiger und kritischer Begleiter technologischer Entwicklung geworden ist.

Steffen Rudolphs vorliegende Dissertation thematisiert die sozialen Ursachen eines ungleichen Zugangs zu sowie Praktiken mit digitalen Medien und behandelt damit ein für die Kommunikationswissenschaft zentrales und aktuelles Thema. Er fragt, ,inwiefern Handlungsmöglichkeiten im Internet - als Zugang und Verwendung - in Abhängigkeit von der sozialen Position stehen und in welchem Maße sich gesellschaftliche Strukturierungen entlang ungleich verteilter Ressourcen entgegen den Individualisierungsannahmen als weiterhin einflussreich auf Lebenschancen und vor allem jenen, die sich mit dem Internet eröffnen - zeigen“" (S. 71).

Rudolph fundiert das Thema Ungleichheit mit einer eindrucksvollen Übersicht theoretischer Zugänge. Er beginnt mit gesellschaftlichen Großentwürfen von Autoren wie Manuel Castells, um die soziale Bedeutung des Zugangs zu digitalen Medien zu diskutieren. Vor diesem Hintergrund führt er in die soziologisch geprägte Forschung zu Ungleichheit ein (Marx, Weber) und arbeitet Konzepte wie Schicht und

Dr. P. Porten-Cheé $(\bowtie)$

Weizenbaum-Institut für die vernetzte Gesellschaft, Berlin, Deutschland

E-Mail: p.porten-chee@fu-berlin.de

Freie Universität Berlin, Berlin, Deutschland 
später Kapital heraus, die für das Verständnis der sozialen Prägung individueller Mediennutzung notwendig sind. Rudolphs Perspektive auf Ungleichheit ist dabei geprägt durch den Ansatz des Habitus (Bourdieu). Diese Sichtweise erlaubt es dem Autor, individuelle Handlungen als in jeder Situation bedingt, gefördert und gehemmt durch die soziale Position einer Person zu beschreiben. Überzeugend legt er dar, wie diese Sichtweise sich von rein individualistischen oder sozio-deterministischen Ansätzen abhebt.

Die Aufarbeitung des Forschungsstands erfolgt auf den ersten Blick umfassend und berichtet die Genese der Digital-Divide-Forschung, beginnend mit der Wissensklufthypothese, über die verschiedenen Modellerweiterungen (First u. Second Digital Divide, Mehrebenenmodelle) bis hin zu einzelnen Faktoren, die für die Erklärung von Digital Divide in der Literatur herangezogen werden (zentral: Bildung, sozioökonomischer Status). Was in diesem Zusammenhang jedoch überrascht, ist der Umstand, dass der Forschungsstand zur Digital Divide und etwa Typologien der Online-Nutzungsforschung auch im Empirie-Teil der Arbeit besprochen werden. Diese Beobachtung führt mich zu einem wesentlichen Kritikpunkt an der Arbeit: dem Aufbau. Nach dem ausführlichen Teil der Theorie und des Forschungsstands läutet Kap. 6 die „Empirische Analyse digitaler Ungleichheiten“ ein. Was verwundert, ist jedoch, dass man sich darin unvermittelt in der Datenauswertung befindet. Ein die empirische Arbeit vorbereitendes Kapitel wäre stattdessen, wie ich finde, aus zwei Gründen notwendig gewesen. Erstens, um vor dem Hintergrund des gesamten Forschungsstands empirisch zu untersuchende Forschungsfragen abzuleiten und gegebenenfalls auch Hypothesen zu formulieren. Zweitens, um die Komplexität (z. B. Habitus als Intersektion zwischen Individuum und Sozialstruktur) von der Theorieebene in die Analyseebene zu überführen, etwa in einem Untersuchungsmodell. In Anbetracht dessen, dass die Digital-Divide-Forschung etabliert ist und der Medienwandel eine differenzierte Nutzung digitaler Medien vorantreibt, hätte ich es begrüßt, die verwendeten quantitativen Daten zum Anlass zu nehmen, eigene Annahmen zu formulieren und zu testen. Ohne einen Abschnitt zur Modellierung fällt es leider schwer, den empirischen Anspruch dieser Arbeit zu bemessen und vor diesem Hintergrund abzuschätzen, inwieweit die berichteten Daten in der Lage sind, die Fragen zu beantworten.

Trotz des problematischen Aufbaus gelingt eine empirische Untersuchung, die es vermag, einen differenzierten Blick auf die sozio-strukturellen Ursachen für Digital Divide zu werfen. Die dafür verwendeten Sekundärdaten sind für die Untersuchungsziele zweckdienlich; die Auswertungsmethoden sind angemessen gewählt und durchgeführt. In mehreren aufeinander aufbauenden Schritten statistischer Datenanalyse zeigt Rudolph, wie stark etwa das Bildungsniveau (immer noch) die Nachrichtenrezeption über digitale Kanäle bedingt, und bringt auch überraschendere Befunde zutage - so etwa, dass Menschen mit geringem ökonomischen Kapital verstärkt das Internet dafür nutzen, ihre strukturellen Defizite zu kompensieren, etwa auf Online-Marktplätzen. An dieser Stelle erweist sich die differenzierte (Faktoren-)Analyse verschiedener Nutzungsarten des Internets als sehr ergiebig.

Zusammenfassend liegt eine Arbeit vor, die ihre Stärke im theoretischen Teil entfaltet, für die sich jedoch aufgrund einer fehlenden Klammer zwischen Theorie und Empirie nur bedingt beurteilen lässt, wie sehr die Arbeit zum Forschungsstand 
über den Digital Divide beiträgt. Dennoch ist die sehr zugängliche Einführung in das Habitus-Konzept sowie die gründliche Aufarbeitung der Divide-Forschung einem breiteren Publikum in unserem Fach zu empfehlen.

Funding Open Access funding provided by Projekt DEAL.

Open Access Dieser Artikel wird unter der Creative Commons Namensnennung 4.0 International Lizenz veröffentlicht, welche die Nutzung, Vervielfältigung, Bearbeitung, Verbreitung und Wiedergabe in jeglichem Medium und Format erlaubt, sofern Sie den/die ursprünglichen Autor(en) und die Quelle ordnungsgemäß nennen, einen Link zur Creative Commons Lizenz beifügen und angeben, ob Änderungen vorgenommen wurden.

Die in diesem Artikel enthaltenen Bilder und sonstiges Drittmaterial unterliegen ebenfalls der genannten Creative Commons Lizenz, sofern sich aus der Abbildungslegende nichts anderes ergibt. Sofern das betreffende Material nicht unter der genannten Creative Commons Lizenz steht und die betreffende Handlung nicht nach gesetzlichen Vorschriften erlaubt ist, ist für die oben aufgeführten Weiterverwendungen des Materials die Einwilligung des jeweiligen Rechteinhabers einzuholen.

Weitere Details zur Lizenz entnehmen Sie bitte der Lizenzinformation auf http://creativecommons.org/ licenses/by/4.0/deed.de.

Dr. Pablo Porten-Cheé ist Leiter der Forschungsgruppe „Digital Citizenship“ am Weizenbaum-Institut für die vernetzte Gesellschaft und Postdoc an der Freien Universität Berlin. 